\title{
Naturalis historia de Plinio: concepción y terapéutica. A propósito de los cuidados de la mujer
}

\section{Plinie's natural history: design and therapeutics. About the care of} women

\section{Naturalis historia Plínio; desenho e terapêutica. Sobre o atendimen- to de mulheres.}

Mercedes Fraile Bravo ${ }^{1}$; Francisco Tirado Altamirano ${ }^{2}$; Jesus Prieto Moreno ${ }^{3}$; Luis Mariano Hernández Neila Edurne Magdaleno Bravo ${ }^{5}$; Luis Sánchez Solís ${ }^{6}$

${ }^{1}$ Licenciada en Enfermera, Licenciada en Antropóloga Social y Cultural. Profesora Filosofía e Historia de la ciencia enfermera. Universidad de Extremadura. Centro Universitario de Mérida.

${ }^{2}$ Licenciado en Enfermería. Profesor Universidad de Extremadura. Centro Universitario de Plasencia

${ }^{3}$ Licenciado en Enfermería y Licenciado en Antropología Social. Profesor Profesor Universidad de Extremadura. Centro Universitario de Plasencia

${ }^{4}$ Profesor Universidad de Extremadura. Centro Universitario de Plasencia

${ }^{5-6}$ Enfermera Asistencial Servicio Extremeño de Salud.

Cómo citar este artículo en edición digital: Fraile Bravo M; Tirado Altamirano F; Prieto Moreno J; Hernández Neila LM; Magdaleno Bravo E; Sánchez Solís L. (2012) Naturalis historia de Plinio: concepción y terapéutica. A propósito de los cuidados de la mujer. Cultura de los Cuidados. (Edición digital) 16, 33. Disponible en: <http://dx.doi.org/10.7184/cuid.2012.33.07>

Correspondencia: Mercedes Fraile Bravo. C/ Santa Joaquina de Jornet. Mérida 06800 Badajoz.

Correo electrónico: mfbravo@unex.es/ Tel. 658989114

Recibido 9/12/2011/ Aceptado: 13/02/2012

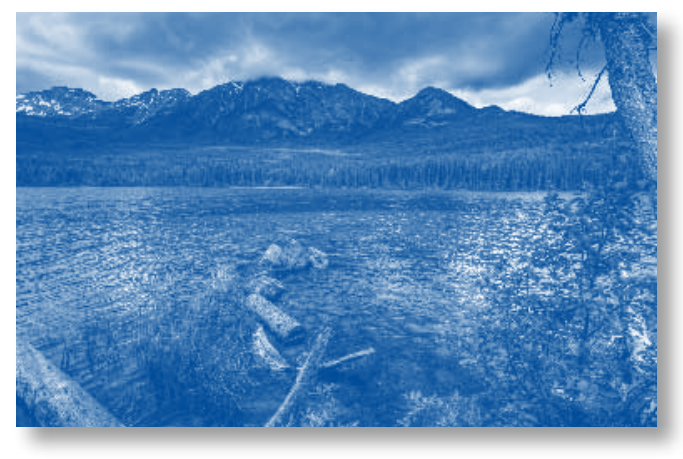

ABSTRACT

The bibliographical sources are basic elements of care study.

The aim of work is the historical reconstruction of caring for women in the Roman era in the early C. I d focusing on the role of design and delivery and the treatment employed.

Using qualitative methodology, historical method, focusing on hermeneutics, we study and analyze the characteristics of the design and the therapy used to alleviate the problems of women in the life cycle of reproduction in Roman times, from a constructivist paradigm

The source used is the Key Pliny's Natural History Second, structured in 37 books. A book dedicated medical XXVI. There are plenty of dedicated therapeutic pharmacopoeia from animals and plants, providing the remedy to be used to facilitate the design, and each of the problems with menstruation, pregnancy and childbirth in women from Roman times

Collecting Roman pharmacopoeia of the early century to C. Use plants and animals and natural elements of therapeutic formula that soothe, heal or cure various health problems of women in particular: conception, pregnancy, childbirth, childbirth and menstruation.

Keywords: History of care, therapeutic wife, Roman 


\section{RESUMO}

As fontes bibliográficas são elementos básicos de estudo cuidado.

O objetivo do trabalho é a reconstrução histórica de cuidar de mulheres na era romana no início dos anos C. I d enfocando o papel do design e de entrega e do tratamento empregado.

Utilizando metodologia qualitativa, método histórico, com foco na hermenêutica, que estudar e analisar as características do design e da terapia utilizada para aliviar os problemas das mulheres no ciclo de vida de reprodução no tempo dos romanos, a partir de um paradigma construtivista

A fonte utilizada é a chave de Plínio Segundo História Natural, estruturado em 37 livros. Um livro dedicado médica XXVI. Há uma abundância de farmacopéia terapêutica dedicada de animais e plantas, fornecendo o remédio a ser usado para facilitar o design, e cada um dos problemas com a menstruação, gravidez e parto em mulheres da época romana

Coleta de Roman farmacopéia do início do século a C. Use plantas e animais e elementos naturais da fórmula terapêutica que aliviar, curar ou curar vários problemas de saúde das mulheres em particular: concepção, gravidez, parto, parto e menstruação.

Palavras-chave: História do cuidado, a esposa de terapêutica, Roman.

\section{RESUMEN}

Las fuentes bibliográficas son elementos básicos para el estudio de los cuidados.

El objetivo de trabajo es la reconstrucción histórica de los cuidados de la mujer, en la época romana, a principios del siglo I d C. centrándolo en la función de concepción y parto y en la terapéutica empleada.
Mediante metodología cualitativa, Método histórico, centrado en la hermenéutica, se estudia y analizan las características de la concepción, así como la terapéutica empleada para paliar los problemas de la mujer en el ciclo vital de la reproducción en la época romana, desde un paradigma constructivista.

La fuente utilizada es la Historia Natural de Cayo Plinio Segundo, estructurado en 37 libros. A medicina se dedican el libro XXVI. Abundan los dedicados a terapéutica y farmacopea a partir de animales y plantas, estableciendo el remedio a utilizar para facilitar la concepción, así como cada uno de los problemas en la menstruación, embarazo y parto en la mujer de la época romana

Recopilación la farmacopea romana de principios del siglo I a C. Utiliza plantas y animales como elementos naturales de formula terapéutica que alivian, sanan o curan diferentes problemas de salud de la mujer en particular: concepción, embarazo, parto, puerperio y menstruación.

Palabras clave: Historia de los cuidados, terapéutica mujer, época romana.

\section{INTRODUCCIÓN Y OBJETIVOS}

El cuidado siempre ha existido desde el comienzo de la humanidad (Tailin, 2011). Siguiendo a Siles en su idea de partida de que los cuidados de salud están influidos por factores históricos-culturales que les dan significado, la historia aparece como una herramienta esencial para el desarrollo del conocimiento de la profesión (Siles, 2010).

Las fuentes bibliográficas han sido y son uno de los elementos básicos para el estudio de los cuidados. De hecho suponen un elemento fundamental en la construcción del conocimiento enfermero en las que se apoya el 
Método Histórico, posibilita el avance del conocimiento humano, ya no solo de los textos escritos sino del mundo simbólico y cultural que el hombre plasma en sus acciones Tamayo Castaño 2011). Así pues, estos dos elementos van a servirnos de soporte para la realización de esta investigación, en la que se erige como fuente principal bibliográfica la Historia Natural de Plinio. La elección de este Manual se realizó de forma casual al encontrar en él información que nos pareció atractiva tras la búsqueda de otro tipo.

Dado que el Manual de Historia Natural de Cayo Segundo Plinio, se sitúa a principios del siglo I de nuestra era, en pleno Apogeo del Imperio Romano, justifica la influencia y la importancia histórico-cultural, como bien sostenía Siles.

Cayo Plinio Segundo, apodo Plinio el Viejo, el Autor, murió en el año 79 a d C, el dia primero de noviembre, cuando con 56 años desempeñaba el cargo de jefe de la escuadra de Tito anclada en Misena. Su inmensa curiosidad le llevó en plena erupción del Vesubio, a la observación directa del fenómeno, como nos relata su sobrino Plinio el joven (Plinio 1999) en las cartas a Tácito, recogidas en este ejemplar. Epoca en la que se iniciaba el receso de la sociedad romana, en el que se presentía la decadencia y el fin de la primera etapa de nuestra civilización occidental, época caracterizada por la pérdida del impulso creador, época en la que se producen los libros de recopilación que almacenan conocimientos sin añadir nada nuevo. Es posible que esta sea la afortunada causa de que se conserven.

El objetivo general de este trabajo es la reconstrucción histórica de los cuidados, y en especial, de los cuidados de la mujer, en la época romana, a principios del siglo I d C. centrándolo en una función exclusiva de la mujer como es la concepción y el parto. Un segundo objetivo estaría encaminado a conocer la terapéutica empleada en los problemas de la mujer

La hipótesis de trabajo manejada es la siguiente: La Historia Natural de Plinio recoge los remedios naturales empleados en los cuidados de los problemas de la mujer.

\section{MÉTODO}

Hemos imprimido a este trabajo un enfoque cualitativo, concretamente el Método Histórico en la firme convicción de que el estudio de la historia de la enfermería constituye un importante pilar para la formación de la identidad profesional. Por tanto, tras un enfoque sistemático, se lleva a cabo la recogida de datos, evaluación crítica de los mismos y la presentación e interpretación de éstos con la consiguiente extracción de conclusiones.

La hermenéutica, como facilitadora de herramientas para comprender a un autor, se nos muestra como el instrumento fundamental.

Utilizando el modelo histórico-estructural que propone Siles (Siles, 2010) estableciendo y analizando las estructuras esenciales de Unidad funcional (Familia)-Marco funcional (Hogar)-Elemento funcional (Profesionales) evolución (Antigüedad), enmarcándose, según estas estructuras en la fase Tribal-Doméstica.

Se estudia y analizan las características de la concepción, así como la terapéutica empleada para paliar los problemas de la mujer en el ciclo vital de la reproducción en la época romana, desde un paradigma constructivista.

La fuente utilizada, es una fuente secundaria, la Historia Natural de Cayo Plinio Segundo, Plinio el Viejo. El Manual de referencia es una edición de especial de 1999 de las Obras Completas de D. Francisco Hernández editadas en Méjico por la Universidad nacional de Méjico en 1976. En los Tomos IV, VI y VII de 


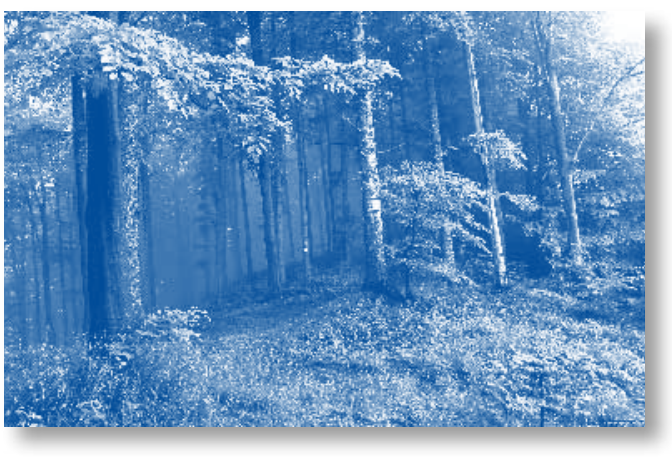

la obra de Hernández, se recoge la Historia Natural de Plinio, concretamente desde los libros primero al vigésimoquinto, siendo transcrito por Jerónimo de Huerta de los libros vigésimo sexto a trigésimoséptimo, en 1624, completando así los treinta y siete libros de que consta la H.N.de Plinio.

El análisis crítico externo de la fuente nos revela su originalidad y la crítica interna (Padilla, 2007; Hernández Conesa 1999)

\section{RESULTADOS}

Se estructura en 37 libros, por materias: geografía, astronomía, botánica, veterinaria, etc. A medicina se dedican el libro XXVI, siendo más abundantes los libros dedicados a la terapéutica y la farmacopea a partir de animales y plantas, estableciendo el tipo de remedio a utilizar para facilitar la concepción, así como cada uno de los problemas en la menstruación, embarazo y parto en la mujer de la época romana.

Los libros XX a XXV conforman un importante tratado de farmacología obtenida de árboles, flores, hierbas, vino, animales de agua,

Nos interesa fundamentalmente la información recogida en los libros, VII, XXVI, XXVII, XXVIII, XXIX y XXX.

1-Libro VII, nos refiere la existencia de partos prodigiosos, en número, la constatación del cambio de sexo y parto de gemelos. Destacan el Capítulo XV.
La menstruación: El Cap XV se refiere a la regla de las mujeres. Se refiere a ella como "La cosa más monstruosa" (esta sangre), de tal manera que el mosto se torna ácido, las mieses que toca no granan, los arboles donde se sientan las mujeres con la regla, se les cae el fruto, los huertos se secan, el lustre de los espejos en que se miran se empañan, el brillo del marfil se empañan, se mueren las colmenas y el cobre y el hierro se tornan en orín y de olor pestilente, altera a naturaleza del Betún de Judea haciéndolo más pastoso e incluso si es ingerida por un perro le desencadena la rabia con mordedura incluida. Prejuicios, mitos y tabúes relacionados con la menstruación que curiosamente han perdurado hasta hace no mucho en nuestra sociedad occidental.

El Libro XXVIII, es su Cap VII, nos ofrece todo un elenco de los poderes de la menstruación, benignos: ahuyentar los granizos y las tempestades, contra las tormentas, sobre todo en navegación. Eso, son los únicos poderes beneficiosos, pues ya comprobamos que en general eran malignos, y sobre todo si coinciden con un eclipse de luna: hacen caer orugas en las mieses, dañan las vides, hacen huir a las abejas de sus colmenas si éstas son tocadas por una mujer menstruando, abortan las yeguas preñadas, por lo que pueden usarse como abortivos incluso entre las mujeres.

Aunque el linimento hecho con sangre menstrual se usa, frente a la gota de los pies en diviesos y erisipelas y tumores planos.

2- El deseo sexual: Libro XXVI, en su Cap $\mathrm{X}$, nos ofrece los remedios sobre "la abstinencia y el apetito de Venus", de tal forma que una infusión de Nimphea heraclia suprime el deseo sexual, los sueños venéreos y la lujuria. Por el contrario, hace aparecer el deseo la raíz del xiphio. Establece cuáles son las plantas que aumentan y cuales disminuyen la libido. 
El estiércol de ratón refrena el apetito venéreo de los hombres (Libro XXIX, Cap XIX)

3- "El mal de las mujeres", fórmulas compuestas por hierbas y leche y/o agua y/o vino, aparecen el Cap XV del libro XXVI, así como los que detienen la regla o incluso ayudan a la expulsión de la placenta ("las pares"), así como el feto muerto. También las que aceleran el parto como la "scordote" bebida.

4- La concepción del sexo: El theligono, que bebido, hace que se conciba un feto con sexo femenino o el "arsenogono" que concibe masculino. (Libro XXVII, Cap X), La raíz de "cinosorche", ingerida por el hombre hace que se engendre varón e ingerida pro la mujer, se engendra hembra”. Existe otra fórmula para engendrar un varón: El hombre y la mujer deben tomar antes de cenar, durante cuarenta días antes de concebir, la raíz de "crateogono", disuelta en la misma cantidad de agua que de vino.

Para recuperar la capacidad concebir y de gestar (Libro XXVIII, Cap XIX), se utiliza los testículos y la sangraza de las liebres al hombre y nueve granos de estiércol de liebre virgen a la mujer, sobre todo para mantener la firmeza de los pechos.

\section{5- Remedios que se toman de las muje-} res: La leche materna por sus características dulces es utilizada como remedio en diversas enfermedades, dependiendo si la mujer ha parido un varón o una hembra, sobre todo si se ha abstenido de alimentarse con vino y de alimentos agrios, sóla o mezclada con clara de huevo, o incluso con miel o incienso. Se empapa una lana con ella para curar los fluxiones de los ojos, y las patologías oculares en general. (Libro XXVIII, Cap VII).

Un capítulo especial a las aplicaciones de la leche de mujer en particular y otros animales en general y de las patologías y supersticiones que cura, en el libro XXVIII, en su Cap IX

\section{CONCLUSIONES}

Este manual nos ofrece un concepto claro de lo que fueron las ciencias naturales desde Aristóteles a los romanos. Supone una importante recopilación de la farmacopea romana de principios del siglo I a C. conservándose íntegramente. Utiliza plantas y animales como elementos naturales de formula terapéutica que alivian, sanan o curan diferentes problemas de salud en general y de la mujer en particular: concepción, embarazo, parto, puerperio y menstruación.

\section{FUENTES Y BIBLIOGRAFÍA}

- Cayo Plinio Segundo (1999). HN Edit Visor (Libro)

-Hernández Conesa, J (1999) Historia de enfermería. Un análisis histórico de los cuidados de enfermería. ED McGraw pag 38-40.(Libro)

- Padilla MI, Borenstein M, Guedes J. Investigación Histórica en la Enfermería: 'posibilidades y metodología. Temperamentum 2007, 5 disponible en http://www.index-f.com/temperamentum/tn5/t1207.php Consultado el 10 de septiembre de 2011

- Siles, J (2010) Historia Cultural de enfermería: reflexión epistemológica y metodológica. Avances de enfermería XXVIII Número especial, 90 años: (120:128) (Libro)

- Tamayo Castaño, E. Los métodos de las Ciencias. www. monografías .com. consultado el 20 agosto 2011

- Tailin Kareli, Nieto, R, Construcción de la epistemología en enfermería. www.monografías.com consultado 10 septiembre 2011. 